\title{
MSFC STREAM MODEL PRELIMINARY RESULTS: MODELING RECENT LEONID AND PERSEID ENCOUNTERS
}

\author{
DANIELLE E. MOSER AND WILLIAM J. COOKE \\ UNITeS, Morgan Research Corp.
}

NASA/Marshall Space Flight Center Building 4487, EV13

Huntsville, AL 35812

E-mail: danielle.e.moser@msfc.nasa.gov

Telephone: 256.544.2423

william.j.cooke@nasa.gov

Fax: 256.544.5754

256.544 .9136

256.544 .5754

Running Head. Modeling LeONID AND PeRSEID ENCOUNTERS 


\title{
MSFC STREAM MODEL PRELIMINARY RESULTS: MODELING RECENT LEONID AND PERSEID ENCOUNTERS
}

\author{
DANIELLE E. MOSER AND WILLIAM J. COOKE \\ NASA/Marshall Space Flight Center, Building 4487 EV13, Huntsville, AL 35812 \\ E-mail:danielle.e.moser@msfc.nasa.gov
}

\begin{abstract}
The cometary meteoroid ejection model of Jones and Brown (1996b) was used to simulate ejection from comets 55P/Tempel-Tuttle during the last 12 revolutions, and the last 9 apparitions of 109P/Swift-Tuttle. Using cometary ephemerides generated by the Jet Propulsion Laboratory's (JPL) HORIZONS Solar System Data and Ephemeris Computation Service, two independent ejection schemes were simulated. In the first case, ejection was simulated in 1 hour time steps along the comet's orbit while it was within $2.5 \mathrm{AU}$ of the Sun. In the second case, ejection was simulated to occur at the hour the comet reached perihelion. A 4th order variable step-size Runge-Kutta integrator was then used to integrate meteoroid position and velocity forward in time, accounting for the effects of radiation pressure, PoyntingRobertson drag, and the gravitational forces of the planets, which were computed using JPL's DE406 planetary ephemerides. An impact parameter was computed for each particle approaching the Earth to create a flux profile, and the results compared to observations of the 1998 and 1999 Leonid showers, and the 1993 and 2004 Perseids.
\end{abstract}

Keywords: Comet, comet ejection, Leonids, meteor, meteor shower, meteoroids; model predictions, numerical integration, Perseids

\section{Introduction}

The Marshall Space Flight Center (MSFC) meteoroid stream model simulates particle ejection and subsequent evolution from comets in order to provide meteor shower forcasts to spacecraft operators for hazard mitigation and mission planning purposes. This paper is concerned with simulating the evolution of the Leonid and Perseid streams associated with comets 55P/Tempel-Tuttle and 109P/Swift-Tuttle, respectively. The model is in a fairly early phase of development, thus the results reported here are preliminary. The immediate aim was to compare the peak solar longitudes resulting from the model with observations of past Leonid and Perseid encounters.

\subsection{OVERVIEW}

\section{Model}

In modeling particle ejection and subsequent evolution from comets Tempel-Tuttle and Swift-Tuttle, the workload is broken into three parts. First the test particles are created, then their positions and velocities are integrated forward in time, and finally the particles are extracted at specific times of interest.

At the first step, the JPL HORIZONS Solar System Data and Ephemeris Computation Service is used to create cometary ephemerides. Particle state vectors are generated for each line in the ephemeris while their physical properties are determined from a uniform, random draw on $\log \beta$, where $\beta$ is the ratio of radiation 
pressure to the Sun's gravitation, and an assumed density. Two separate ejection schemes (two sets of state vectors) are simulated: Timestep and Perihelion. Ejection is simulated in 1 hour time steps along the comet's orbit while it is within $2.5 \mathrm{AU}$ of the Sun, hereafter referred to as the Timestep case. Ejection occurring only at the hour of perihelion passage is referred to as the Perihelion case. The model of Jones and Brown (1996b) is used to determine particle ejection velocity. The particle velocity far from the comet is given by

$$
V_{\infty}=41.7(\sin (\alpha / 2))^{0.37}(\cos z)^{0.519} R_{c}^{1 / 2} m^{-1 / 6} \rho^{-1 / 3} r_{h}^{-1.038}
$$

where $\alpha$ is the semi-angle of the spherical cap of ejection, $\mathrm{z}$ is the local solar zenith angle, $R_{c}$ is the comet radius, $m$ is the particle mass, $\rho$ is the particle density, and $r_{h}$ is heliocentric distance. Sublimation is taken to occur on the day side of the comet within a constrained cap angle $(\alpha)$; for further description of the cap angle and the other parameters, the reader is referred to Jones and Brown (1996b) and Jones (1995). Other studies (e.g. Brown and Jones, 1998; Göckel and Jehn, 2000; Welch, 2003) have investigated this ejection velocity model.

At the second step, a 4th order variable step-size Runge-Kutta integrator is used to integrate meteoroid position and velocity forward in time, accounting for the effects of radiation pressure, Poynting-Robertson drag, and the gravitational influences of 7 planets, Venus through Neptune. JPL's DE406 is used to compute the positions of the planets; interpolation is done via cubic spline. Integration of the particle ensemble is performed on 9 dedicated AMD Athlon ${ }^{\mathrm{TM}}$ 64-bit FX processors. The CPU time for each case, as discussed in Section 2.2, is approximately 5 days for the Leonids and 2 weeks for the Perseids.

In the last step, particles are extracted within $0.01 \mathrm{AU}$ of Earth within 1 week before and after the expected shower peak. The node-Earth distance for each particle is computed, along with an impact parameter (IP) for each meteoroid approaching Earth. The impact parameter is defined as follows

$I P=\left(R_{E}+h_{\text {atmos }}\right) / D$

where $R_{E}$ is the radius of the earth, $h_{a t m o s}$ is the height of the atmosphere, and $D$ is the Earth-particle distance at nodal crossing; it is scaled to 1 at the top of the atmosphere. It is known that D is not the closest a particle will approach Earth, especially in the case of streams associated with low inclination comets. In the case of streams associated with high inclination comets, however, the difference between D and the actual closest approach distance is inconsequential. Thus the IP is valid for streams with large orbital inclinations, as is the case for the Leonids and the Perseids.

\subsection{INPUTS}

In modeling the Leonids it was necessary to simulate ejection from comet TempelTuttle during the last 12 revolutions, epochs 1600-1965. Three hundred thousand particles were ejected during each epoch, in both ejection schemes, for a total of 3.6 million particles for each case, Timestep and Perihelion. The meteoroid production rate was varied with the heliocentric distance as $r_{h}{ }^{-5}$, based on previous simulations, and $\beta$ ranged from $\sim 10^{-5}$ to $10^{-2}$, resulting in a mass range between approximately 1 $\mu \mathrm{g}$ and $1 \mathrm{~kg}$, as the density was assumed to be $1000 \mathrm{~kg} \mathrm{~m}^{-3}$. A cap angle of $\alpha=30^{\circ}$ was chosen. The radius of the comet was assumed to be $2.0 \mathrm{~km}$ (Hainaut et al., 1998). 
For each ejection case and two different cap angles, comet Swift-Tuttle was modeled with 900,000 particles ejected over each of its last 9 revolutions dating from 826 to 1862 . This resulted in 4 total Perseid cases with 8.1 million particles each. Particle production depended on $\mathrm{r}_{\mathrm{h}}^{-6}$ (Fomenkova et al., 1995). Again, $\beta$ ranged from $\sim 10^{-5}$ to $10^{-2}$, the corresponding masses from $1 \mathrm{~kg}$ down to $1 \mu \mathrm{g}$, assuming density was $1000 \mathrm{~kg} \mathrm{~m}^{-3}$. Cap angles of $25^{\circ}$ and $60^{\circ}$ were chosen based on the literature, respectively Jones (1995), and Jones and Brown (1996a) and Brown and Jones (1998). The radius of the comet was assumed to be $11.0 \mathrm{~km}$, based on Boehnhardt et al. (1996).

\subsection{MODEL COMPARISON}

Per Welch (2003), numerical simulations of meteor showers tend to conform to one of two types whereby: 1) large numbers of meteoroids are ejected from a parent comet and the subsequent evolution of these particles is followed until the period of interest, or 2) the meteoroid orbit with the correct change of period necessary to pass through the relevant node at the time of the shower in a given year is determined for a given ejection epoch. The MSFC model is of the first type. It is similar to models seen in Brown (1999), Brown and Arlt (2000), Göckel and Jehn (2000), and Vaubaillon and Colas (2002) in that respect. The models in Kondrat'eva and Reznikov (1985), Wu and Williams (1996), Asher (1999), and McNaught and Asher (1999) are of the second type. Welch (2003) is a combination of these types. It is unknown at this time under which type category the work of Lyytinen (1999) and Lyytinen and Van Flandern (2004) falls.

A brief summary comparing the Leonid MSFC model to the Leonid models of other authors is given in Table I. The main categories of comparison involve how the cometary ephemerides are created, the ejection velocity law used, the particle production dependence on heliocentric distance, the ejection scheme - particles ejected along the comet's orbit or only at perihelion, and the integrator used to integrate the position of the comets back in time and/or evolve the particles forward in time. The MSFC model uses JPL Horizons to calculate the cometary ephemerides, instead of introducing the additional complexity of integrating the comets, whose dynamics are significantly influenced by jetting, reflected in the A1 and A2 terms. Ejection velocity is based on Jones and Brown (1996b) and Jones (1995). Type 1 models, with the exception of Vaubaillon and Colas (2002), and Welch (2003) have explored this ejection velocity model, among others, with a set cap angle of $60^{\circ}$. Vaubaillon and Colas (2002) and Welch (2003) have investigated the ejection velocity model of Crifo and Rodionov (1997). The MSFC model's particle production dependence on heliocentric distance is not fixed at $r_{h}{ }^{-2}$ but is instead based on observational evidence found in the literature or determined based on previous simulations. Two different ejection schemes are compared in this model: particle ejection along the comet's orbit within $2.5 \mathrm{AU}$ and also ejection just at the hour of perihelion, like models of Types 1 and 2. In the MSFC model a 4th order variable step-size Runge-Kutta integrator is used to evolve the particle orbits, like Brown (1999), and Brown and Arlt (2000). Various other numerical integrators have been used, including Runge-Kutta-Nystrom 12(10)17 (e.g. Welch, 2003), Radau 15 (e.g. Vaubaillon and Colas, 2002), and Stumpff-Weiss (e.g. Göckel and Jehn, 2000). 


\section{Results and Discussion}

A subset of the total number of particles propagated, those extracted within $0.01 \mathrm{AU}$ of Earth within 1 week before and after the expected shower peaks of interest, is now examined. As stated previously, an impact parameter (IP) is computed for each particle of this set approaching the Earth. In effect, this is the scaled probability that the particle will hit Earth. (It must be noted that a stream that approaches Earth, but does not appear to cross its path, still has a nonzero probability of striking the Earth.)

The particle IPs are summed in $0.01^{\circ}$ solar longitude bins (this corresponds to a time interval of 14.6 minutes), thereby representing cumulative probabilities. Ideally, the IPs would be summed in $0.005^{\circ}$ bins, corresponding to the Earth moving approximately 1 Earth diameter plus the height of the atmosphere. There were not enough particles in the subset, however, for this binning scheme, so $0.01^{\circ}$ solar longitude bins were used.

A Lorentzian is fit to the binned IP versus solar longitude - essentially the flux profile - in order to determine the time of the shower peak. The results are compared to observations of the 1998 and 1999 Leonid showers, and the 1993 and 2004 Perseids. In this way, the goal - to compare the solar longitudes of the main shower peak resulting from the model with observations of past Leonid and Perseid encounters, thereby validating the MSFC meteoroid stream model's timing - is achieved.

In the following sections the results of this validation are described. Cross-section plots showing the composition of the showers by stream are included and appear as parts (a) of Figures 1-4. Each of the particles in parts (a) has an associated IP. These IPs are summed in solar longitude bins and, for comparison purposes the amplitudes are scaled, thus creating a flux profile, shown in parts (b) of Figures 1-4. The model results are simply scaled to the peak ZHR observed so that the time of the observed peak and that of the peak predicted by the model can be easily compared. The observational data is also shown in parts (b). Finally, predictions from other modelers, where possible, are given in tabular form.

\subsection{LEONIDS}

Figure 1 shows the modeling results of the 1998 Leonids. The shower had two main peaks. The first peak, thought to be characterized by old ejecta according to Arlt and Brown (1999), was not modeled. The second peak (the nodal peak), however, is clearly prominent at solar longitude $235.311^{\circ} \pm 0.007^{\circ}$ (ibid). It is made up of particles ejected from recent passages of Tempel-Tuttle, namely 1965, 1932, and 1899. The MSFC model predicted a peak time at $235.27^{\circ} \pm 0.01^{\circ}$, a difference of about 1 hour. Table II summarizes the results of the 1998 Leonid encounter alongside predictions from other authors.

The 1999 Leonid result can be seen in Figure 2. A storm of activity was observed at $235.285^{\circ} \pm 0.001^{\circ}$ (Arlt et al., 1999). The MSFC model predicted the storm peak, consisting mainly of particles from 1899 and 1932 but also including those from 1965 , at $235.282^{\circ} \pm 0.002^{\circ}$. This is a difference of just under 2 minutes. The MSFC results for the 1999 Leonids, and the results of other authors, are presented in Table III. 


\subsection{PERSEIDS}

Figure 3 illustrates the modeling results of the 1993 Perseids. The main peak occurred at $139.53^{\circ} \pm 0.01^{\circ}$ (Bone and Evans, 1996; Rendtel and Brown, 1997). A time of $139.491^{\circ} \pm 0.002^{\circ}$ was determined from the MSFC model, a difference of 57 minutes. Particles ejected from Swift-Tuttle's passages in 1862, 1479, and 1610 were the main contributors. This breakdown is somewhat different from Brown (1999). The 1862 stream contributed the most significantly.

The 2004 Perseid result is shown in Figure 4. The maximum occurred at $139.443^{\circ} \pm 0.003^{\circ}$ (Arlt, unpublished observations). The MSFC model predicted a peak time of $139.42^{\circ} \pm 0.01^{\circ}$, a difference of 34 minutes. Particles from 1862 made up the peak.

Table IV summarizes the results of the Perseid encounters.

\subsection{MODEL VARIABLES}

Two different ejection cases, Timestep and Perihelion, were simulated for both the Leonids and the Perseids. In general, the Perihelion case produces narrower particle distributions, as is to be expected. An example of this can be seen for the 2000 Leonids in Figure 5(a). These two cases yield similar peak times at this preliminary analysis stage. It is the Timestep case, however, that is felt to be more physically realistic.

The Leonids were only simulated with one cap angle value. For the Perseids, two different cap angles, $\alpha=25^{\circ}$ and $\alpha=60^{\circ}$, were tested. The smaller of the two values simulates an exit cone of about $60^{\circ}$; the $60^{\circ}$ cap angle corresponds to a hemispherical exit cone (Jones and Brown, 1996b). Of the two values, the $60^{\circ}$ cap angle model generally predicts a solar longitude closer to that of the observed maximum ZHR. The 1998 Perseids, shown in Figure 5(b), is a good example of this. With scaling, the maximum is clearly distinguishable despite the scatter. The observed peak ZHR occurred at solar longitude $139.75^{\circ} \pm 0.03^{\circ}$ (Arlt, 1999). The $60^{\circ}$ and $25^{\circ}$ cap angle models have peaks at $139.72^{\circ} \pm 0.01^{\circ}$ and $139.83^{\circ} \pm 0.01^{\circ}$, respectively.

\section{Summary}

The MSFC stream model predicts, within about an hour or better, the peak times of several Leonid and Perseid encounters. Further refinement is necessary in analyzing the results, especially concerning ZHR calculations. Work exploring the effects of ejection schemes, the Timestep case and the Perihelion case, and cap angles is underway. Additionally, the inclusion of observational data, such as population indices, is underway in order to better constrain parameters associated with particle ejection from comets. Future work addressing the effects of different cometary ephemerides is planned; these effects are expected to be significant.

\section{Acknowledgements}

This work was supported by NASA contract NNM04AA02C. The authors also wish to acknowledge the IMO; a great number of their compiled observations were used as bases of comparison. 
Arlt, R.: 1999, IMO 27, 237-249.

\section{References}

Arlt, R. and Brown, P.: 1999, IMO 27, 267-285.

Arlt, R., Rubio, L. B., Brown, P., and Gyssens, M.: 1999, IMO 27, 286-295.

Asher, D. J.: 1999, Mon. Not. R. Astron. Soc. 307, 919-924.

Boehnhardt, H., Birkle, K., and Osterloh, M.: 1996, Earth, Moon, Planets 73, 51-70.

Bone, N. M. and Evans, S. J.: 1996, J. Br. Astron. Assoc. 106, 33-39.

Brown, P.: 1999, 'Evolution of Two Periodic Meteoroid Streams: The Perseids and Leonids', Ph.D.

Thesis, Univ. of Western Ontario.

Brown, P. and Arlt, R.: 2000, Mon. Not. R. Astron. Soc. 319, 419-428.

Brown, P. and Jones, J.: 1998, Icarus 133, 36-68.

Crifo, J.F.: 1995, ApJ 445, 470-488.

Crifo, J. F. and Rodionov, A. V.: 1997, Icarus 127, 319-353.

Fomenkova, M. N., Jones, B., Pina, R., Puetter, R., Sarmecanic, J., Gherz, R. and Jones, T.: 1995, Astron. J. 110, 1866-1874.

Göckel, C. and Jehn, R.: 2000, Mon. Not. R. Astron. Soc. 317, L1-L5.

Hainaut, O. R., Meech, K. J., Boehnhardt, H., and West, R. M.: 1998, Astron. Astrophys. 333, 746-752.

Jones, J.: 1995, Mon. Not. R. Astron. Soc. 275, 773-780.

Jones, J. and Brown, P.: 1996a, in B. A. S. Gustafson and M. S. Hanner (eds.), Physics, Chemistry, and Dynamics of Interplanetary Dust, APS Conference Series 104, 105-108.

Jones, J. and Brown, P.: 1996b, in B. A. S. Gustafson and M. S. Hanner (eds.), Physics, Chemistry, and Dynamics of Interplanetary Dust, APS Conference Series 104, 137-140.

Kondrat'eva, E. D., and Reznikov, E. A.: 1985, Sol. Syst. Res. 19, 96-101.

Kulikov, D. K.: 1960, Byul. Inst. Teor. Astron. 7, 770-797.

Lyytinen, E.: 1999, Meta Research Bulletin 8, 33-40.

Lyytinen, E. and van Flandern, T.: 2004, IMO 32, 51-53.

McNaught, R. H. and Asher, D. J.: 1999, IMO 27, 85-102.

Nakano, S.: 1998, Minor Planet Circ., 31070.

Rendtel, J. and Brown, P.: 1997, Planet. Space Sci. 45, 585-593.

Rocher, P.: 2002, www.bdl.fr/ephem/comets/HTML/english/Comete_e.html

Vaubaillon, J. and Colas, F.: 2002, in Proceedings of Asteroids, Comets, Meteors (ACM 2002), 181-184.

Welch, P. G.: 2003, Mon. Not. R. Astron. Soc. 342, 971-994.

Wu, Z. and Williams, I. P.: 1996, Mon. Not. R. Astron. Soc. 280, 1210-1218.

Yeomans, D. K., Yau, K. K., and Weissman, P.R.: 1996, Icarus 124, 407-413.

\section{Figure Captions}

Figure 1. Results of the 1998 Leonids, Timestep case. (a) Cross-section in x-y ecliptic coordinates showing the composition of the shower by stream. As shown in the legend, the different symbols represent various streams by year, and the solid black line represents the Earth's path. Ejecta from 1899, 1932, and 1965 played the greatest role in the nodal peak. (b) Comparing the scaled model with observations. Observational data was collected by the International Meteor Organization (IMO) and published in Arlt and Brown (1999). The model is scaled to the maximum ZHR of the nodal peak.

Figure 2. Results of the 1999 Leonids, Timestep case. (a) X-Y cross-section plot showing the composition of the shower by stream. As shown in the legend, the different symbols represent various streams by year, and the solid black line represents the Earth's path. Revs 2 (1932) and 3 (1899) played the greatest role in the storm peak, as well as particles from 1965. (b) Comparing the scaled model with observations. Observational data was collected by the IMO and published in Arlt et al. (1999)

Figure 3. Results of the 1993 Perseids, Timestep case, 60 cap angle. (a) Cross-section in x-y ecliptic coordinates showing the composition of the shower by stream. As shown in the legend, the different symbols represent various streams by year, and the solid black line represents the Earth's path. Ejecta from years 1862, 1479, and 1610 made up the bulk of the peak, with 1862 being most prominent. (b) Comparing the scaled model with observations. Observational data was collected by the British Astronomical Association (BAA) and published in Bone and Evans (1996).

Figure 4. Results of the 2004 Perseids, Timestep case, $60^{\circ}$ cap angle. (a) X-Y cross-section plot showing the composition of the shower by stream. As shown in the legend, the different symbols represent various streams by year, and the solid black line represents the Earth's path. The maximum was made up from the 
1862 stream. (b) Comparing the scaled model with observations. Observational data was collected by the IMO, and is not yet published (Arlt, unpublished observations).

Figure 5. (a) X-Y cross-section plot showing the composition of the shower by stream for the 2000 Leonids. As shown in the legend, the different symbols represent various streams by year, and the solid black line represents the Earth's path. The Perihelion ejection case (in black) is shown superimposed on the Timestep case (in gray). Note especially how focused the 1965 and 1932 streams of the Perihelion case are, as compared to the Timestep case. (b) Comparing two scaled models with observations for the 1998 Perseids. The $60^{\circ}$ cap angle model (in gray) matches the time of the observed maximum better than the $25^{\circ}$ model (in black). Observational data was collected by the IMO and published in Arlt (1999). 


\section{Tables}

Table I

\begin{tabular}{|c|c|c|c|c|c|}
\hline Authors (Leonids) & $\begin{array}{l}\text { Cometary Elements/ } \\
\text { Ephemerides Source }\end{array}$ & Ejection Velocity & $\begin{array}{c}\text { Particle } \\
\text { Production } \\
\text { Dependence }\end{array}$ & Ejection Scheme & Integrator \\
\hline MSFC & JPL Horizons & $\begin{array}{l}\text { Jones ejection (Jones, 1995; Jones } \\
\text { and Brown, 1996b) }\end{array}$ & $\mathrm{r}_{\mathrm{h}}^{-5}$ & $\begin{array}{l}\text { Timestep, within } 2.5 \\
\text { AU; Perihelion }\end{array}$ & Runge-Kutta 4 \\
\hline $\begin{array}{l}\text { Brown (1999), } \\
\text { Brown \& Arlt (2000) }\end{array}$ & Yeomans et al. (1996) & $\begin{array}{l}\text { Jones ejection (Jones, 1995), } \\
\text { modified Jones and parabolic Jones } \\
\text { (Brown \& Jones, 1998), Crifo (1995) }\end{array}$ & $r_{h}^{-2}$ & Timestep, within $4 \mathrm{AU}$ & Runge-Kutta 4 \\
\hline Göckel \& Jehn (2000) & - & $\begin{array}{l}\text { Jones ejection (Jones, 1995), } \\
\text { modified Jones and parabolic Jones } \\
\text { (Brown \& Jones, 1998), Crifo (1995) }\end{array}$ & $\mathrm{r}^{0}$ & Timestep, within $4 \mathrm{AU}$ & Stumpff-Weiss \\
\hline Vaubaillon \& Colas (2002) & Rocher (2002) & Crifo \& Rodionov (1997) & $\begin{array}{l}\mathrm{r}^{0}, \text { weighted } \\
\text { later by } \mathrm{r}_{\mathrm{h}}^{-2}\end{array}$ & Timestep, within $3 \mathrm{AU}$ & Radau 15 \\
\hline Kondrat'eva \& Reznikov (1985) & independent & $\begin{array}{l}\text { set at } 1 \text { value at perihelion }-0 \mathrm{~km} / \mathrm{s} \text { - } \\
\text { then iteratively determined }\end{array}$ & $\mathrm{n} / \mathrm{a}$ & Perihelion & $\begin{array}{c}\text { algorithm of } \\
\text { Kulikov (1960) }\end{array}$ \\
\hline Wu \& Williams (1996) & $\mathrm{n} / \mathrm{a}$ & $\begin{array}{l}\text { set at } 1 \text { value at perihelion - a mean } \\
\text { ejection velocity less than } 0.6 \mathrm{~km} / \mathrm{s}\end{array}$ & $\mathrm{n} / \mathrm{a}$ & Perihelion & Runge-Kutta-Nystrom \\
\hline $\begin{array}{l}\text { Asher (1999), } \\
\text { McNaught \& Asher (1999) }\end{array}$ & $\begin{array}{l}\text { Nakano (1998), } \\
\text { Yeomans et al. (1996) }\end{array}$ & $\mathrm{n} / \mathrm{a}$ & $\mathrm{n} / \mathrm{a}$ & Perihelion & Radau 15 \\
\hline Welch (2003) & Nakano (1998) & $\begin{array}{l}\text { Jones ejection (Jones, 1995), } \\
\text { modified Jones (Brown \& Jones, } \\
\text { 1998), Crifo \& Rodionov (1997) }\end{array}$ & $r_{h}^{-2}$ & $\begin{array}{c}\text { Timestep, within } 3.5 \\
\text { AU }\end{array}$ & Runge-Kutta-Nystrom \\
\hline
\end{tabular}


Table II

\begin{tabular}{|c|c|c|c|c|c|c|}
\hline Year & Peak & $\begin{array}{c}\text { McNaught } \\
\text { \& Asher } \\
\text { (1999) }\end{array}$ & $\begin{array}{l}\text { Brown } \\
\& \text { Arlt } \\
(2000)\end{array}$ & $\begin{array}{l}\text { MSFC } \\
\text { Result }\end{array}$ & $\begin{array}{l}\text { Observ- } \\
\text { ations }\end{array}$ & $\begin{array}{c}\text { MSFC } \\
\text { Difference }\end{array}$ \\
\hline 1998 & Nodal & 235.26 & $23 \overline{3}-\overline{34}$ & $\begin{array}{r}235.265 \\
-235.262\end{array}$ & $\begin{array}{c}235.311 \\
n / a\end{array}$ & $=\underset{\mathrm{n} / \mathrm{a}}{\sim 1.1 \mathrm{hr}}$ \\
\hline & $\begin{array}{l}1932 \\
1890\end{array}$ & 235.27 & 235.22 & $\begin{array}{l}235.263 \\
235\end{array}$ & $\mathrm{n} / \mathrm{a}$ & $\mathrm{n} / \mathrm{a}$ \\
\hline
\end{tabular}

Table III

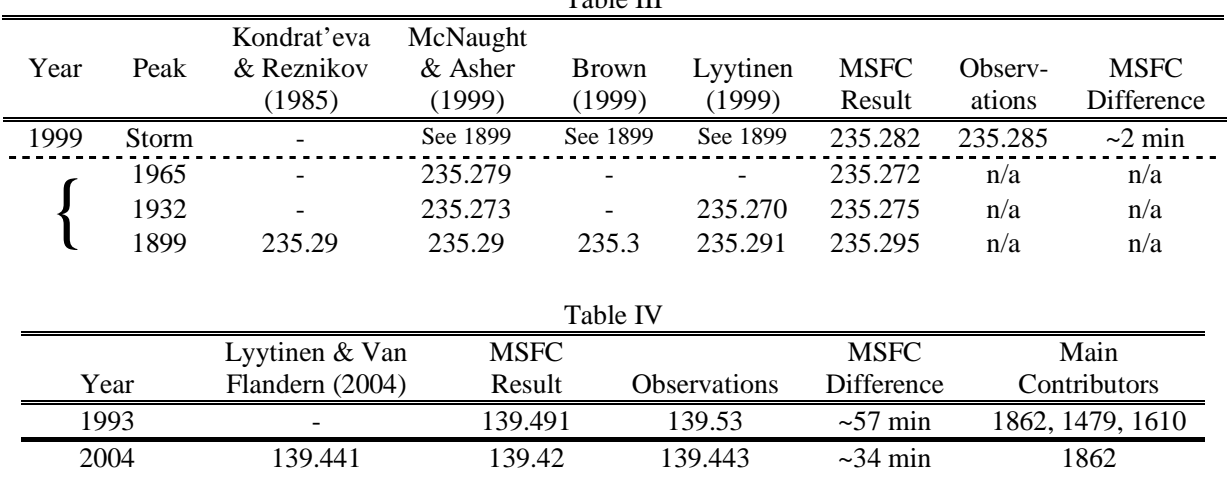

Table I. A brief summary comparing the Leonid MSFC model to the Leonid models of other authors. The main categories of comparison involve how the cometary ephemerides are created (most modelers take cometary elements from various sources and integrate backwards), the ejection velocity law used, the particle production dependence on heliocentric distance, the ejection scheme - particles ejected along the comet's orbit or only at perihelion, and the integrator used to integrate the position of the comets back in time and/or evolve the particles forward in time. The top section of the table shows models of Type 1, the middle section shows models of Type 2, and the bottom section shows the hybrid. It is unknown at this time under which type category the work of Lyytinen (1999) and Lyytinen and Van Flandern (2004) falls; these works are not included in this table.

Table II. This table shows the modeling results of various authors alongside the results of the MSFC model and the observations for the 1998 Leonids. The activity peak near the passage of the descending node of Tempel-Tuttle is referred to as the nodal peak; the fireball peak made up of older ejecta was not modeled. Modeling by McNaught and Asher (1999) and Brown and Arlt (2000) found that the 1965 and 1932 streams contributed to the nodal peak and calculated separate solar longitudes for each. Binning the IPs of ejecta from different epochs separately and fitting a curve to the data, the solar longitudes of the maxima for 1965 and 1932 ejecta from the MSFC model are shown for comparison purposes. Material from 1899 was also found to be a contributor and shown accordingly. Observations showed a peak at solar longitude 235.311 (Arlt and Brown, 1999). An analysis of the subset described in the text, combining ejecta from each perihelion passage (1965, 1932, and 1899 included) gives a peak at 235.265, a difference of about an hour. Errors are omitted for convenience.

Table III. This table shows the modeling results of various authors alongside the results of the MSFC model and the observations for the 1999 Leonids. A storm of activity was observed at solar longitude 235.285 (Arlt et al., 1999). In the models of Kondrat'eva and Reznikov (1985), McNaught and Asher (1999), Brown (1999), and Lyytinen (1999), as well as the MSFC model, the major (or only) contribution to the storm peak came from particles ejected in 1899. Solar longitudes of the maxima for material ejected in 1899, 1932, and 1965 are given separately. Binning the IPs of ejecta from different epochs individually and fitting a curve to the data, the solar longitudes of the maxima for the 1899-1965 streams from the MSFC model are shown for comparison purposes. An analysis of the subset described in the text, combining ejecta from each perihelion passage $(1965,1932$, and 1899 included) gives a storm peak at 235.282, a difference of about 2 minutes. Errors are omitted for convenience. 
Table IV. This table shows the modeling results the MSFC model, alongside the results of Lyytinen \& Van Flandern (2004) where possible, and the observations for the 1993 and 2004 Perseids. The main peak of the 1993 Leonids was at 139.53 (Bone and Evans, 1996; Rendtel and Brown, 1997). The MSFC model showed a peak at 139.491, a difference of about 57 minutes. Particles ejected from Swift-Tuttle's passages in 1862, 1479, and 1610 were the main contributors. In 2004, material from 1862 was the main contributor to the peak. The prediction of Lyytinen and Van Flandern (2004) was very close to what was observed. The result of the MSFC model, with a peak at 139.42, was about 34 minutes premature. Errors are again omitted for convenience. 
Figure 1
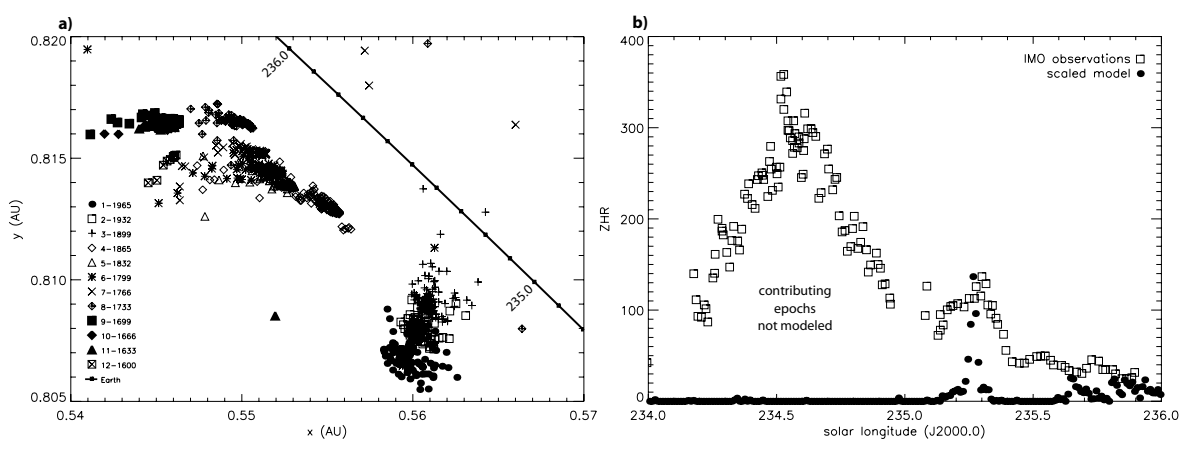

Figure 2
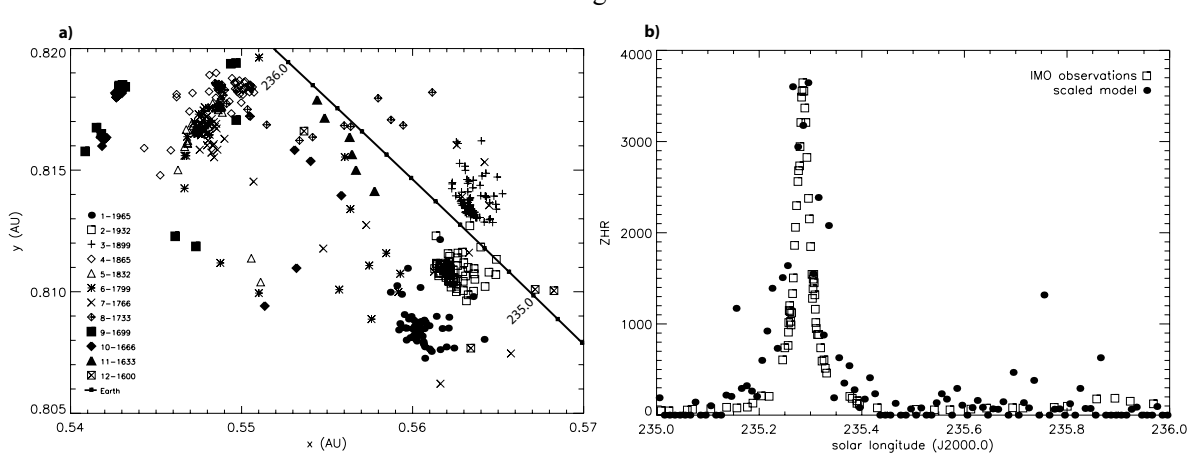

Figure 3
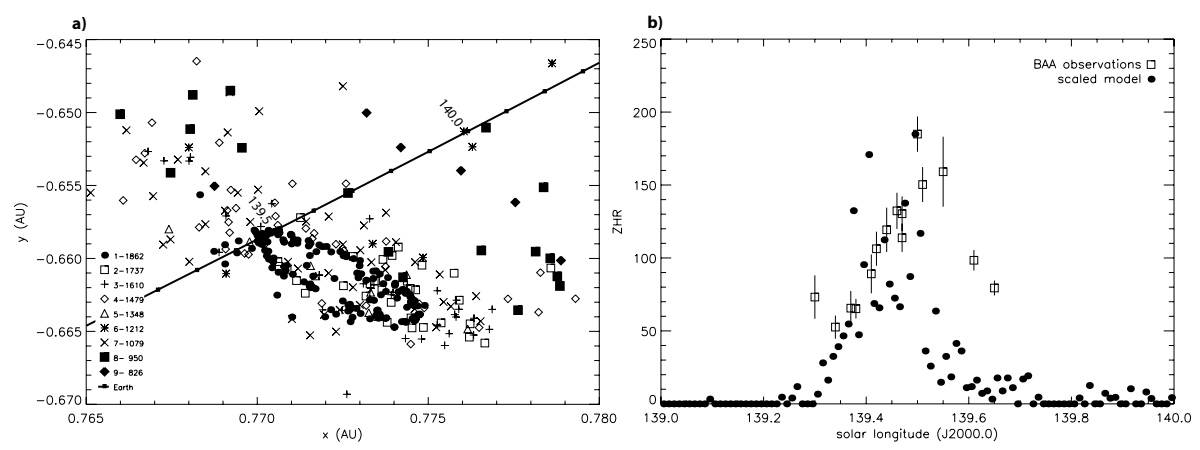
Figure 1
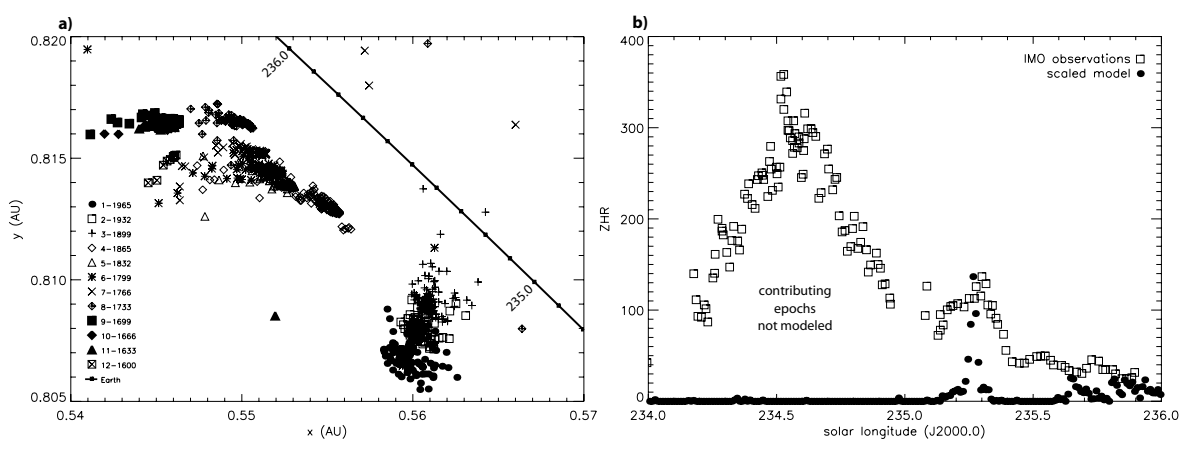

Figure 2
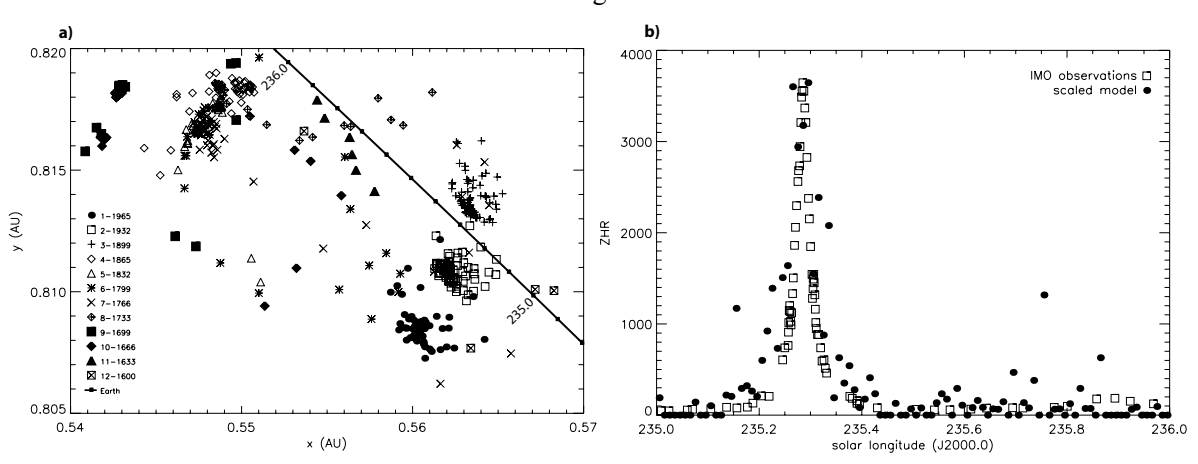

Figure 3
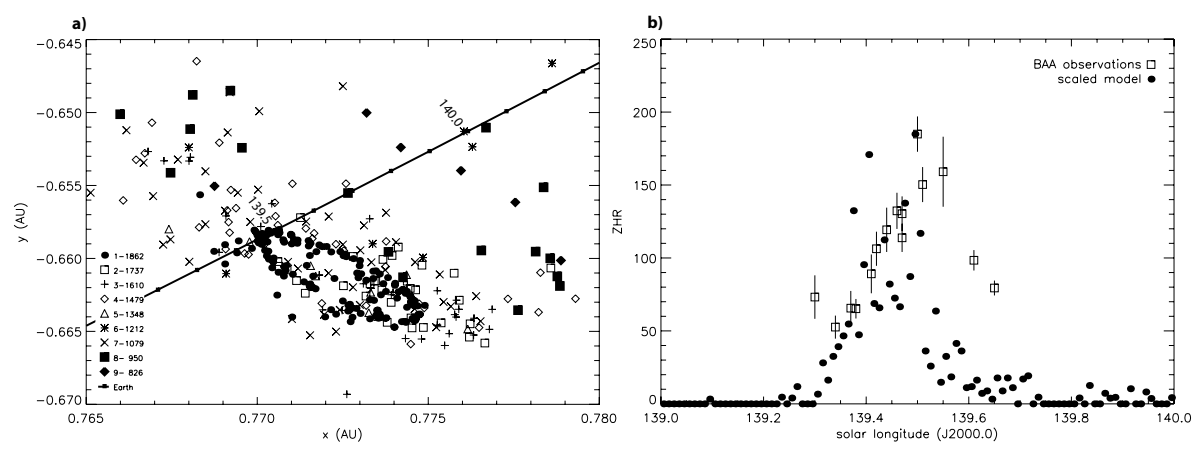
Figure 4
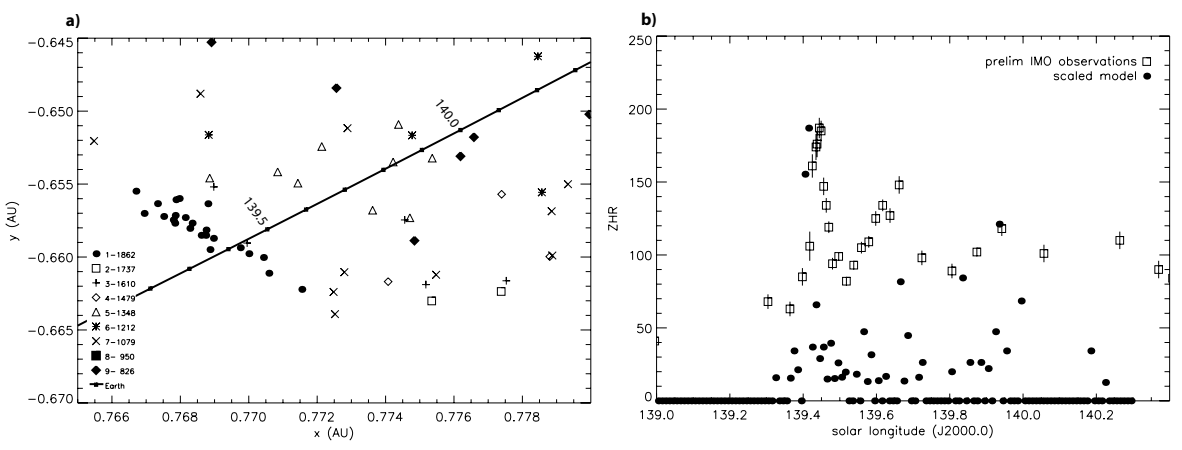

Figure 5
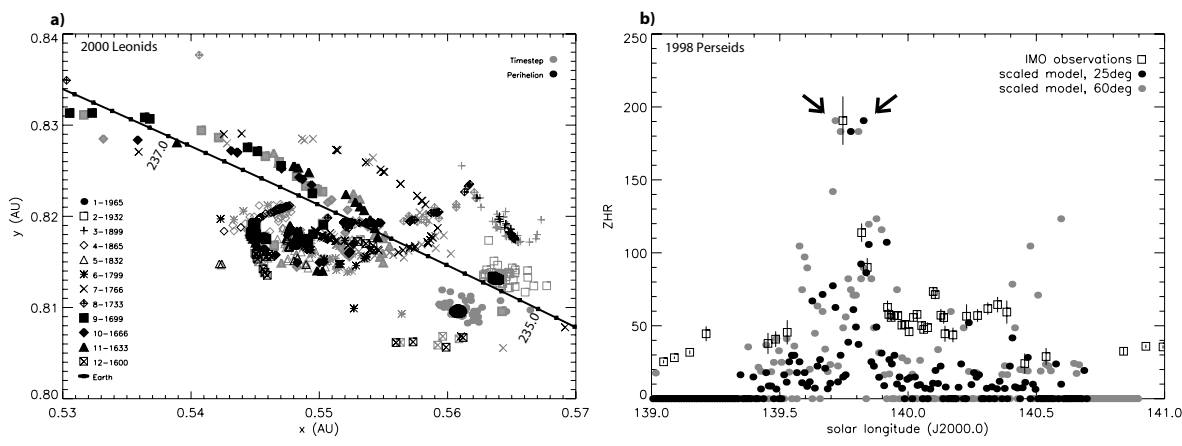